\title{
Finite element simulation of high-speed cutting of nickel-based alloy
}

\author{
Yong YANG ${ }^{1,2, a}$, Yuwen GUO ${ }^{1}$, Fengyu WANG ${ }^{3}$ \\ ${ }^{1}$ Chinese Research Academy of Environmental Sciences in Beijing, China \\ ${ }^{2}$ Qingdao Technological University in Qingdao, China \\ ${ }^{3}$ Qingdao new world environmental protection limited liability company in Qingdao, China
}

\begin{abstract}
By analyzing microstructure of the material, a finite element model of high-speed cutting process more close to the practical instance was put forward. The microstructure of nickel-based alloy was obtained based SEM experiment, and the digital model of microstructure was built. Based on above study, finite element model of highspeed cutting of nickel-based alloy integrating macro and micro physical characters was established. Further, finite element simulation and analysis of high-speed cutting of nickel-based alloy were conducted, and the saw-tooth chip, cutting force variation curve and cutting temperature field distribution pictures were got. Research shows that grain boundary occur serious distortion at chip and tool contact area during saw-tooth chip forming, and the grain boundary structure changes will cause the change of cutting force during generating adiabatic shear band. So reducing cutting force and improving the processing quality can be achieved by changing the internal microscopic structure of workpiece.
\end{abstract}

\section{Introduction}

Nickel-based alloy material is a kind of widely applied in the aviation materials [1]. However, due to coefficient of thermal conductivity of nickel-based alloy material is small, high temperature, high chemical activity in the process of cutting easily tend to forms alloy with knives, making a kind of typical difficult-to-machine materials [2-3]. In recent years, with the continuous improvement of computer performance, finite element simulation technology becomes a new mean to high speed cutting process of the refractory materials [4-5]. Christian et al simulated the serrated chip formation process of Inconel 718 using ABAQUS/Explicit grid adaptive function [6]; Lv Shaoyu investigated cutting process of nickel-based superalloy GH4169 using AdvantEdge finite element analysis software, and the machined surface temperature, strain and strain rate distribution were got, whose research showed that higher temperature and higher strain, strain rate appeared surface contact area for nickel-based alloy GH4169 [7]; Zhou Jun studied the changing rule of the adiabatic shear band inside the serrated chip through the experimental during high-speed cutting nickel-based alloy GH4169 [8].

However, the current finite element simulation researches mainly focus the macrostructure of material, and the simulation research considering material microstructure characteristics is rare. In view of this situation, this study carried out finite element modeling and simulating research based on the microstructure characteristics of nickel-based alloy, which will provide theoretical guidance for the research of qualitative

\footnotetext{
a Corresponding author: yyong901@163.com
}

relations between microstructure and macro cutting performance.

\section{Digital Modeling of Microstructure}

The SEM (scanning electron microscope) experiment for nickel-based alloy GH4169 was carried out. The alloy annealed at high temperature, and was made a sample of $10 \mathrm{~mm} \times 10 \mathrm{~mm}$ through wire electro discharge. Further, the sampling, grinding, polishing and etching processing were conducted. Sample cutting process is shown in Figure 1.

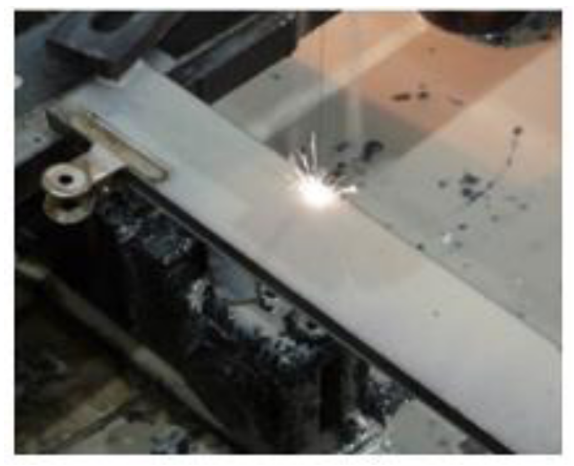

Figure 1. Wire electro discharge process.

After polishing processing, the alloy sample was fixed in the sample stage with conductive adhesive and put into vacuum chamber of scanning electron microscope. The obtained SEM images are shown in Figure 2. The 
distribution rule of grain boundary can be clearly seen from the SEM images.

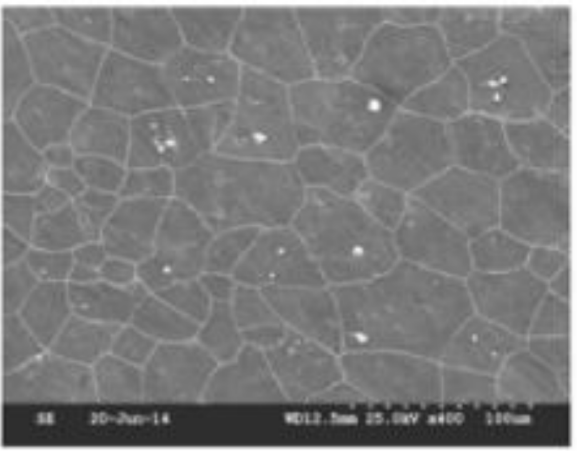

Figure 2. SEM images of microstructure.

According to the SEM image, the grain boundary of nickel-based alloy was obvious, and the black background of the degree of differentiation was very good. The images in the graph contained plenty of salt pepper noise after dealing with the median filter to eliminate noise. Finally, the morphological image processing was conducted and the grain boundary node data was obtained, as shown in Figure 3.

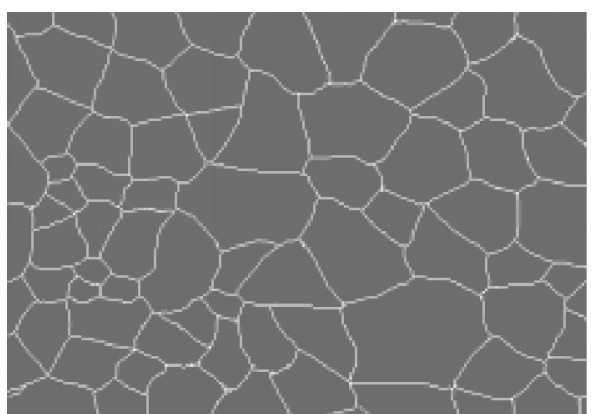

Figure 3. Nickel-based alloy microstructure digital model

\section{Finite element model of high-speed cutting of nickel-based alloy}

Based on digital model of microstructure of nickel-based alloy, using ABAQUS finite element simulation software, the finite element model of workpiece was established. The length of the model was $0.25 \mathrm{~mm}$, the height was 1 $\mathrm{mm}$, the cutting cambium thickness was $0.54 \mathrm{~mm}$, and the failure layer thickness was $0.004 \mathrm{~mm}$. The model was meshed with four nodes thermal coupling plane strain unit (CPE4RT), and the workpiece was divided into 7130 units, tool was 4586 units. The finite element model of high-speed cutting considering macrostructure and microstructure is shown in Figure 4.

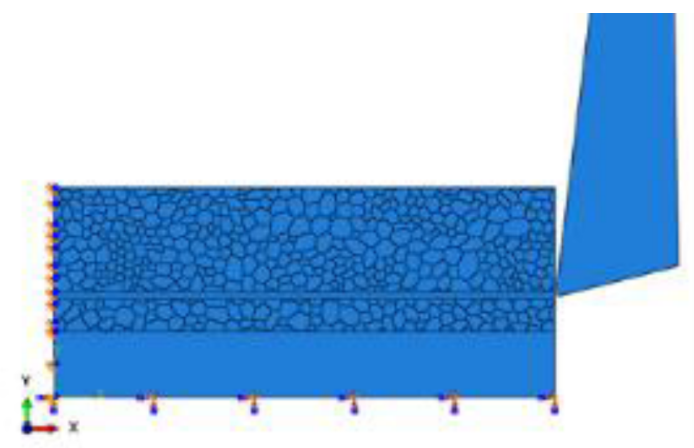

Figure 4. Finite element model of high-speed cutting

The tool was taken as reference point, due to the high speed cutting range of was $30 \sim 200 \mathrm{~m} / \mathrm{min}$, so the speed of reference point was set to $110 \mathrm{~m} / \mathrm{min}$. The tool material was cemented carbide, and rake angle was $0^{\circ}$, clearance angle was $20^{\circ}$. The finite element simulation of the workpiece length and cutting parameters are shown in Table 1.

Table 1. Cutting parameters used in finite element simulation.

\begin{tabular}{|c|c|}
\hline Cutting parameters & Quantitative value \\
\hline Cutting speed v $(\mathrm{m} / \mathrm{min})$ & 110 \\
\hline Feed per tooth $f_{z}(\mathrm{~mm})$ & 0.5 \\
\hline Axial depth of out $a_{p}(\mathrm{~mm})$ & 1.5 \\
\hline Radial depth of cut $a_{e}(\mathrm{~mm})$ & 1.5 \\
\hline
\end{tabular}

\section{Finite element simulation and analysis}

Figure 5 shows serrated chip formation process of nickelbased alloy. It can be seen from the diagram that the grain boundary structure in the adiabatic shear zone happened serious distortion. On the contact surface of the chip and tool, there was a serious distortion of grain boundary, with which the local microstructure transformation and grain crushing can be predicted.

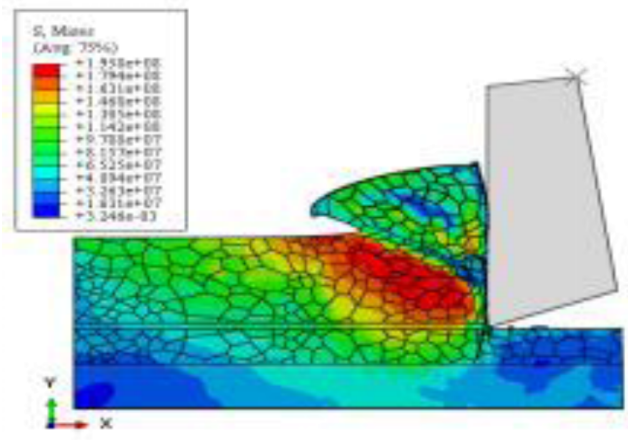

(a) Formation of the first serrated chip 


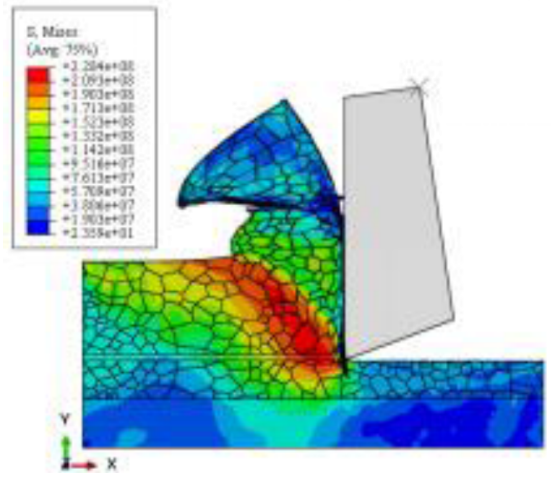

(b) Formation of the second serrated chip

Figure 5. Serrated chip formation process of nickel-based alloy

Figure 6 shows cutting force curve in $\mathrm{X}$ direction of finite element simulation. From this picture, it can be seen that there was a total of four peaks and troughs. During generating process of each zigzag agglomerate, the cutting force increased gradually in early stage, and when thermoplastic instability happened, the adiabatic shear band formed and the cutting force gradually declined.

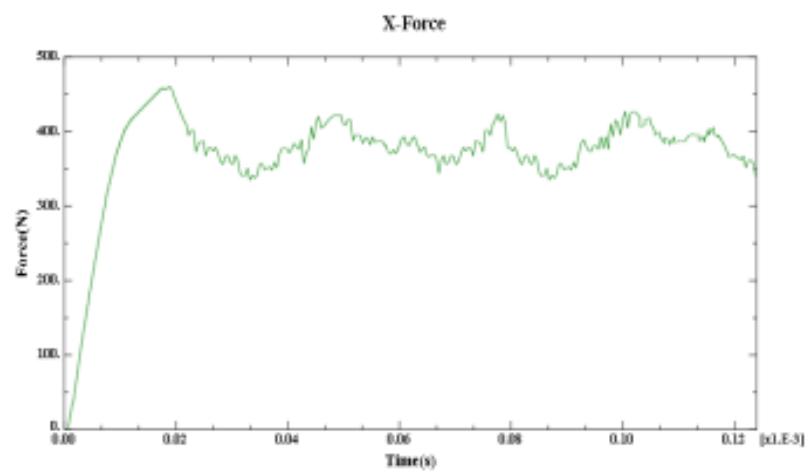

Figure 6. Cutting force curve in $\mathrm{X}$ direction

Figure 7 shows cutting force curve in $\mathrm{Y}$ direction of finite element simulation. It can be seen from the graph that the curve also presented four troughs, which further proved that the fluctuation of cutting force was caused by adiabatic shear band. By analyzing the serrated chip formation process, it can be concluded that the change of grain boundary structure in the process of generating adiabatic shear band will cause the change of cutting force. So the fluctuation of cutting force can better be reduced by changing the internal microstructure organization of workpiece, and the machining quality and tool life can also be improved.

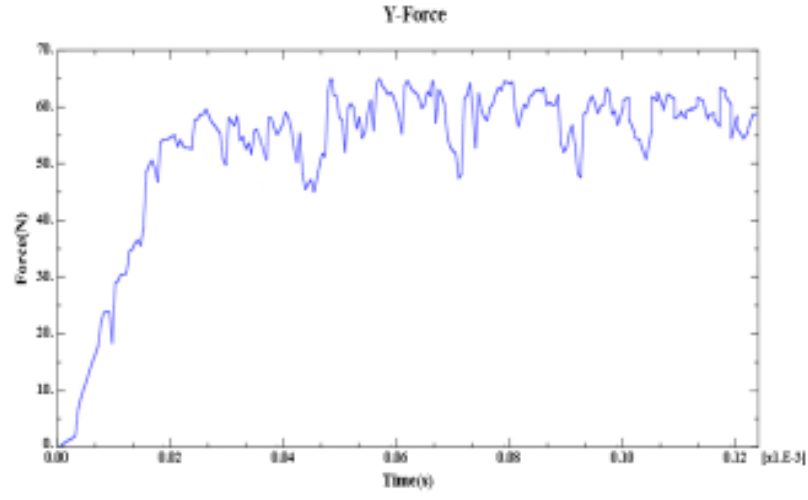

Figure 7. Cutting force curve in $\mathrm{Y}$ direction

Figure 8 shows temperature field distribution of workpiece and tool during high-speed cutting process. Due to the time of cutting process is short, the quantity of heat in the workpiece spreads with smaller scale. Due to specific heat and thermal conductivity of cutting tool is large, the heat in the cutting tool has a wide distribution range. It can be seen from the diagram, cutting temperature in shear zone is higher, which directly led to the chip thermoplastic instability occurs, then produce the adiabatic shear band.

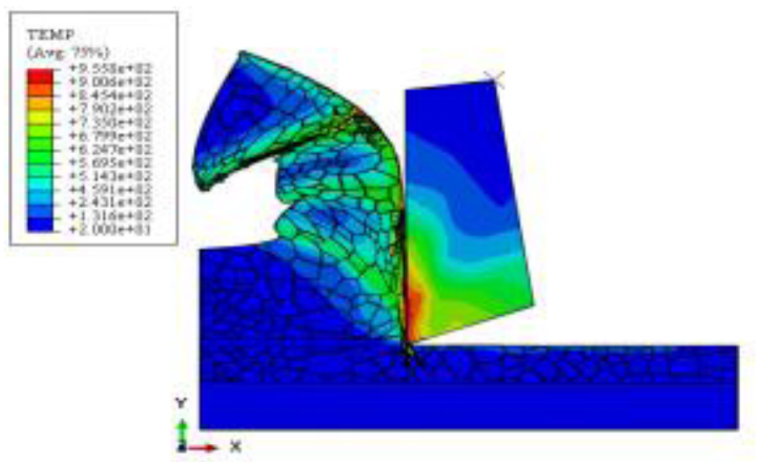

Figure 8. Temperature field distribution of nickel-based alloy workpiece and tool

\section{Conclusion}

(1) Starting from the microstructure of materials, through SEM experiment and its image processing, the digital model of nickel-based alloy microstructure was established; Then, using the ABAQUS finite element software, finite element model of high-speed cutting process considering macro and microstructure characteristic was constructed; Based on the above research, finite element simulation of high-speed cutting of nickel-based alloy was conducted. The serrated chip formation process, the change rule of cutting force curve and the cutting temperature field distribution were obtained.

(2) Tradional finite element simulation of high speed cutting focus on the macroscopic geometric characteristics of the material, while this research in this paper introduces the characteristics of material microstructure, which can get closer to the actual working condition and details. This work will help to consummate the study in this field. 
(3) Further, the finite element model can be used to select optimal machining parameters and improve tool design, and excessive and expensive trial and error experiment process can be avoided.

\section{Acknowledgments}

This work was supported by the China Postdoctoral Science Foundation under Grant Nos. 2014M550783.

\section{References}

1. D.G. Thakur, B. Ramamoorthy, L. Vijayaraghavan, Materials and Design, 30, 1718(2009)

2. E.G. Ng, D.W. Lee, CIRP Annals-Manufacturing Technology, 49, 41 (2001)

3. S. Luca, G.F. Marria, International Journal of Machine Tools\&Manufacture, 18, 815 (2008)

4. Y. Yang, M. Li, K.R. Li, The International Journal of Advanced Manufacturing Technology, 70, 1803(2014)

5. D. Yahya, A. Ersan, C. Necip, Journal of Materials Processing Technology, 171, 1(2006)

6. H. Christian, S. Bob, Journal of Materials Processing Technology, 186, 66(2007)

7. L.V. Shaoyu, Shandong University, (2013)

8. J. ZHOU, Harbin institute of technology, (2012) 